\title{
Laser Boost Of A Small Interstellar Ram Jet To Obtain Operational Velocity. Implications For The DM Rocket / Ram Jet Model
}

\author{
Andrew Walcott Beckwith ${ }^{\mathrm{a}}$ \\ ${ }^{a}$ American Institute of beam energy propulsion, \\ abeckwith@uh.edu, beckwith@aibep.org
}

\begin{abstract}
In other conference research papers, Beckwith obtained a maximum DM mass/ energy value of up to $5 \mathrm{TeV}$, as opposed to $400 \mathrm{GeV}$ for $\mathrm{DM}$, which may mean more convertible power for a dark matter ram jet. The consequences are from assuming that axions are CDM, and KK gravitons are for WDM, then $\rho_{\text {Warm-Dark-Matter }}$ would dominate not only structure formation in early universe formation , but would also influence the viability of the DM ram jet applications for interstellar travel. The increase in convertible DM mass makes the ram jet a conceivable option. This paper in addition to describing the scientific issues leading to that 5 TeV mass for DM also what are necessary and sufficient laser boost systems which would permit a ram net to become operational
\end{abstract}

Keywords: axions, seed lasers, DM, ram jet, Lagrange points

PACS: 42.62.-b, 90. +n, 95.35. +d

\section{INTRODUCTION - HOW DARK MATTER INTER RELATES WITH LASER PROPULSION}

The author is aware that Physics and Engineering specialists use very different terminology. The purpose of this document is to get physics researchers to seriously consider the light sail, and some foundational laser engineering problems, and to push Engineers into seriously thinking of Dark matter, as a potential power source. Since the two audiences are different, there will inevitably be those who claim that no paper can satisfy either audience. Hence, this document which will attempt to push specialists to obtain new background. The author's interest in the problem, especially of Dark Matter was revived in the 12 Marcel Grossman meeting.

Before bringing up what is to be said about the laser system, it is appropriate for a list of inquiry questions, prior to reading the paper. On page 4 of this document is a list of three force equations. Which one will be chosen affects the delta $\mathrm{V}$ which could be used for laser assisted space travel, as well as the power needed for utilization of adequate force hitting a space craft. That can only be tested in near earth orbit. Once the appropriate space tests are done, the next order of business would be in determining what power a laser would have to be set at L4 and L5 Lagrange points for travel up to the Kuiper belt by laser power. Also, YAGS were chosen as a stable platform for known laser generation. If more than about 100 Giga Hertz power for say 
up to a month of usage, then a completely new Laser system would have to be developed. The problem of the Dark matter ram jet at or after the Kuiper belt is reached involves another generation of power, which is reflected in equation (6) below, as a momentum transfer. This last part is for now speculative, and it is appropriate to state the line of inquiry which leads to possibly using $5 \mathrm{TeV}$ top mass values for Dark matter as due to $\mathrm{KK}$ gravitons and the $\mathrm{KK}$ graviton tower construction.

When at the 12 Marcell Grossman meeting, July $200917^{\text {th }}$, the author talked with Roszkowski ${ }^{1}$, at the Paris Observatory as to what would happen to DM if hot and cold DM models were mixed together.., Roszkowsk ${ }^{1}$ stated there would be no structural changes which would occur in galaxy formation, if two cold DM candidates would be partially mixed. Conversely, Roszkowsk ${ }^{1}$ referred to significant formation and density fluctuation changes if warm and cold DM candidates were mixed together. The author assumes a mix of DM types, as far as the interstellar mix, which will have consequences for the DM ram jet. Next. Having settled upon looking at the KK graviton as a dark matter candidate, the author settled upon using

$m_{n}($ Graviton $)=\frac{n}{L}+10^{-65}$ grams, with the lowest rest mass being determined with $n$ equal to zero, and the highest version of graviton mass as of $5 \mathrm{TeV}$. The index $\mathrm{n}$ for the graviton refers to an additional dimension, above four dimensions, whereas $\mathrm{L}$ is the rough diameter of the additional spatial dimension. This increased mass will factor into our discussion of the ram jet. To understand what will be done, the paper will first delve into why the rocket equation implies inefficient, costly space travel, then go into the three stages of a space fairing trip of a probe to another star system. Then, to distinguish between different DM candidates, it briefly delves into the reason for the choice of a KK graviton for a DM candidate. The chosen new top level candidate mass, of $5 \mathrm{TeV}$ is convenient, since it provides much more change in momentum, as seen in equation 4 below. In addition, if there is a mix of both warm and cold dark matter candidates, with warm DM represented by KK graviton 'towers' and axions representing cold $\mathrm{DM}^{2}$, then there may be enough abundance of $\mathrm{DM}$ in inter galactic space to serve as the 'fuel' for a upgraded 'DM' ram jet.

\section{First, The Rocket Equation. I.e. Why Boost To Near Earth Orbit Are Expensive With Chemical Rockets, Plus Light Sails For Propulsion}

The rocket equation and the low exhaust velocity of chemical fuels are at the root of the high cost. Laser boosting of space crafts are a way about this problem. In a different context, laser boosting will be done to obtain up to $1 / 20^{\text {th }}$ of the speed of light, as a pre cursor to having the DM ram jet kick in.

$$
\Delta v=v_{e} \cdot \ln \frac{m_{0}}{m}
$$

This can be presented as having $\mathrm{m}_{0}$ as the initial total mass, including propellant. One also can have $\mathrm{m}_{1}$ as the final total mass., and having as a stated given $v_{\mathrm{e}}$ as the effective exhaust velocity. 


$$
v_{e}=I_{s p} \cdot g_{0}
$$

Also, $\Delta v$ is the delta- $v$. Where as $m_{0} / m_{1}$ is the mass ratio. The following table illustrates the problem ${ }^{3}$

\begin{tabular}{|c|l|l|}
\hline Table 1 : & mass ratio $m_{0} / m_{1}$ & versus $\Delta v$ \\
\hline 1 & 0 \\
\hline 2 & 0.693 \\
\hline 3 & 1.098 \\
\hline 4 & 1.386 \\
\hline 5 & 1.609 \\
\hline$\ldots .$. & 33 & 3.496 \\
\hline
\end{tabular}

It is obvious from inspection as to why laser boost to Earth orbit have been considered. Chemical rockets, as Henson wrote to the author ${ }^{3}$ in a private communications are very inefficient. The AIBEP meeting will bring up what can be said about such technology. For the authors demonstration, it is appropriate to consider the problem of laser assists for boosting a space craft in the regions in near space conditions, and to obtain conditions for up to $1 / 20^{\text {th }}$ the speed of light velocity being reached, so a DM ram jet can begin to operate. The reason why laser assisted ram jets have been considered as a way to have space crafts go to other near by stars is because the best current rocket technology can do is about $30 \mathrm{~km} / \mathrm{s}-0.01 \%$ of light speed. That's 10,000 years per light-year in travel time, about the average speed of stars passing each other in the galaxy. For use of a photon assisted drive to get to another star, say 4 light years away, using low boost technology, up to a million years would be needed. If one wants to have interstellar probes going to another star in a century of travel time, different engineering technologies not based upon chemical rockets or plasma rockets such as VASMIR need to be used ${ }^{4}$.

\begin{tabular}{|l|l|l|l|l|}
\hline Table 2 - getting there & \multicolumn{2}{l|}{} \\
\hline System & Velocity (km/s) & $\begin{array}{l}\text { Velocity } \\
\text { (lightspeed= 1.0) }\end{array}$ & $\begin{array}{l}\text { years per light- } \\
\text { year }\end{array}$ & $\begin{array}{l}\text { To Alpha } \\
\text { Centauri (years) }\end{array}$ \\
\hline Current rockets & 30 & 0.0001 & 10,000 & 44,000 \\
\hline VASIMR & 300 & 0.001 & 1,000 & 4,400 \\
\hline solar sail MAX & 1,000 & 0.0033 & 300 & 1,320 \\
\hline Orion(Dyson) & 10,000 & 0.033 & 30 & 132 \\
\hline
\end{tabular}

If an engineer wishes a light sail propulsion system for a space craft, the engineer would design for, either ground or space based laser systems hitting a light sail. Note that Space vehicles can be made much lighter and smaller if they do not have to carry their source of power. Power can be supplied through lasers projected on structures called 'light sails.' The sail material could be some form of Mylar - both thin and strong. Steering the sail and aiming the huge lasers, however, are not trivial problems. By huge lasers, the author is thinking of 10 Giga watts shining on a 1 kilometer in diameter sail just to send a 16 gram payload to the closest star. The laser beam must be precisely aimed on target for as long as possible to get the desired velocities. Realistically though, the laser beams would only work hitting a light sail for space 
craft with a wail travel within a small fraction of a light year. It would then be appropriate to think of how to obtain up to $1 / 20^{\text {th }}$ the speed of light, via this method, and then perhaps think of employing a DM ram jet.

\section{Breaking up portions of the trip into three stages}

(1) Moving from Earth orbit to Mars orbit

The author views either a ground based laser system hitting a light sail, or using VASMIR, as a plasma rocket boost to Mars orbit, as optimal to getting the space craft out to Mars orbits.. The lasers would have to be based upon Earth itself; and five to six of them roughly spaced along the equatorial regions would be enough to supply a powered light pressure venue to push the space craft. The preferred venue would be perhaps to use lasers similar to what was presented as an Yb: YAG $200 \mathrm{~kW}$ laser system as outlined by Sherstobitov, et al. ${ }^{5}$. As indicated in Sherstobitov, et. al. ${ }^{4}$, figure 5 , relative beam size spreading is not a serious problem, as well as atmospheric turbulence would for an Earth to Mars trip be a not insurmountable problem as far power transfer to a Earth to Mars orbit trip. The problem would be in getting five to six coordinated laser sites to be effectively administered by a scientific body. IMO, politics, not scientific feasibility is the limiting factor If Earth bound lasers would be impacted by politics as a significant administrative issue, and then the VASMIR boost system to Mars would perhaps be the preferred venue.

(2) Traveling from Mars, to the Kuiper belt

Use of lasers placed in the Lagrange points about the Earth-Moon system. This supposition is the authors own, due to the necessity of minimizing the impact of rotation of either the Earth or Moon on a sail for propulsion of the space craft to the Kuiper belt. Note that Lagrange points L4 and L5 constitute stable equilibrium points, so that an object placed there would be in a stable orbit with respect to the Earth and Moon. With small departures from L4 or L5, there would be an effective restoring force to bring a satellite back to the stable point. The L4 and L5 points make equilateral triangles with the Earth and Moon.

(3) Post Kuiper belt travel, i.e. time to employ the DM rocket

\section{Getting first to Mars orbit, alternate procedures, before using laser at the L4 and L5 Lagrange points powering a light sail}

First would be to use VASMIR, as a way to get a space craft to Mars Orbit. For those who wish to know what VASMIR is, the following engine diagram suffices to make the point. Secondly, if the plasma rocket VASMIR were not doable as an engineering project, would be to use five or more earth bound lasers, in the equatorial regions , roughly equally spaced to , on a rotating basis, to push a light sail powered space craft out to Mars orbit. 


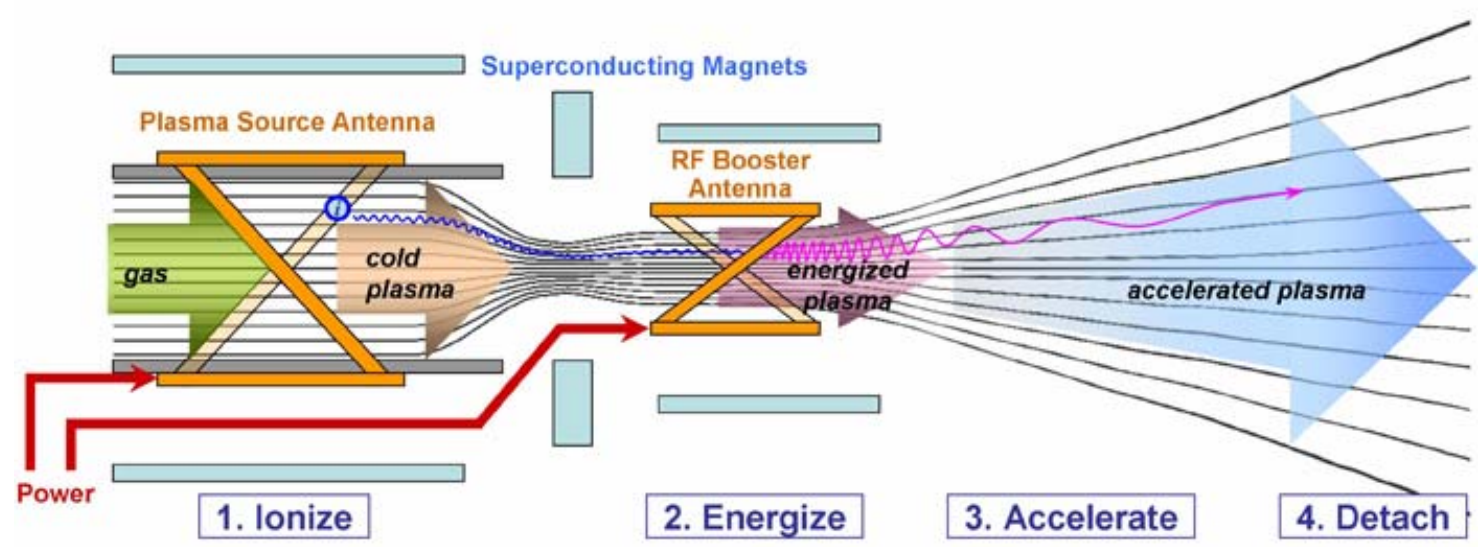

FIGURE 1. Standard working diagram of VASLOV concept

Force equation considerations, and the light sail.

From Millis ${ }^{6}$, the following variations, with $\mathrm{P}$ pressure from a laser hitting a light sail of area $\mathrm{A}$, and with a fudge factor of $\delta$ put in, in the case of real Radiometers, taking into account what could be expected in terms of sail material properties, and sail geometry, plus the degree energy impinging upon the sail has been locally altered, reciprocally across the front and back of the sail. As Millis ${ }^{6}$ writes it, for force upon the sail

$$
F \equiv \frac{P}{A}-\text { Differntial }- \text { Sail : Similar to an ideal Radiometer vane }
$$

$F \equiv\left(\frac{\delta^{2}-1}{\delta}\right) \cdot \frac{P}{A}-$ Induction - Sail : Similar to real Radiometer vane

$F \equiv 2 \cdot \frac{P}{A}-$ Diode - Sail : Similar to a one way mirror

Details as to the fudge factor of $\delta$ being put in can only be resolved via space vacuum tests of a laser- sail system in near earth orbit.

\section{Obtaining A Working 10 Giga Watts Laser (The Real Laser Systems At L4 And L5 Lagrange Points Would Be More Powerful)}

The Giga watt Laser, as described by Y. Satov, ${ }^{7}$ et al (2004) is a $\mathrm{CO}_{2}$ SKATE and its output would appear to be able to approach ten Giga watts. The problem is that the laser system for hitting a space craft would have to be operational for quite a long time, enough so that the space craft would be able to get out to the Kuiper belt. The Kuiper Belt is a disk-shaped region past the orbit of Neptune extending roughly from 30 to 50 AU. One would likely need a system like a High Power Nd: YAG Laser, with a variant of the YAG laser, and that would lead to considerable engineering challenges. One of them would be to develop seed lasers for 
the YAG Laser ${ }^{8}$. Note that highly stable low power seed laser plays a crucial role in these systems by keeping the high power, Q-switched, Nd:YAG laser at a fixed wavelength and single mode. In addition, the seed laser reduces shot-to-shot intensity fluctuations due to mode beating in the YAG laser, which cause damage to internal optics and reduce the lasers lifetime. Current seed lasers are large, expensive, and suffer under vibration and field use. ${ }^{8}$ The problem would be in obtaining and developing a seed laser which combined optical Bragg waveguide structures and new semiconductor laser technology to create a narrow line width laser at the precise wavelength. After the seed laser development would be the even bigger problem. Secondly, if the lasers were to be put to the Lagrange L4 and L5 point, would be the support and logistics problems A full accounting of what would be required for appropriate seed laser for a variant of a stable powerful YAG Laser awaits engineering development work. The detail of finding an appropriate seed laser is brought up as a concept to be developed which requires specific laser $\mathrm{R}$ and $\mathrm{D}$ work. I.e. sufficiently stable YAG lasers capable of a boost of a space craft up to the KUIPER belt requires break through technology as far as appropriate seed lasers, which would probably be the source of a DARPA style initiative. The author is aware of the complexity of seed lasers, as a way to stabilize YAG lasers, and also picked a space position in the L4 and L5 Lagrange points of the Earth-Moon system among other things to obtain a working background which would facilitate cooling of the laser. I.e. having a high power laser operate for extended time period leads to virtually unworkable head disposal problems and issues for the laser system in the presence of a thick atmosphere. In addition, the L4 and L5 Lagrange Earth - Moon points were picked as a spatial position for laser firing on a sail which is free of the complexity of rotation of either the Earth or Moon, as a platform issue for how to aim the laser to a laser sail.

\section{Hierarchy of engineering issues for getting lasers operational}

First of all, is finding an appropriate seed laser system which would help stabilize a YAG style laser system for long term usage. Philip Battle of Bozeman, Montana ${ }^{8}$ has filed for patents as far as stabilizing YAG laser systems. In addition, his proposal for seed lasers was the recipient of a NASA grant in $2002{ }^{8}$. The author is of the opinion that his proposal is currently very crude, but promising and needs considerable expansion and development. Secondly, if a ground based system, as opposed to getting VASMIR to boost a space craft to MARS orbit is to be used, again assuming a YAG style system of the form discussed by Sherstobitov ${ }^{4}$ et al (2004), one needs near orbit space tests to determine the $\delta$ for a real induction sail. I.e. theorizing about it will not work. I.e. the author will later contact Philip Battle, of MSU ${ }^{8}$, and others of a similar background to begin the hard task of finding appropriate seed lasers for stabilization of a candidate YAG laser system, of sufficient power.

Now for the DM rocket / ram jet problem, as proposed. As put in, in a discussion by Beckwith, 2009, for SPESIF, 2009

Quoting from the 2009 DM conference paper by A.W. Beckwith ${ }^{9}$ (2009):” So, we can only talk about perhaps a ram jet engineering construction, i.e., scooping up 
Axions /DM from the interstellar void and using that as a fuel source. So how do we get around this? As can be inferred from P. Sikivie ${ }^{10}$, "Every axion which is converted to a photon with the same total energy and going in the same direction produces a momentum kick of

$$
\Delta p=m c \times \gamma \cdot(1-\beta)
$$

Where $\mathrm{m}$ is the axion rest mass." What is the rest mass of a KK DM graviton candidate? It is up to a mass of $5 \mathrm{TeV}$. The conversion factor to be considered is 5 $\mathrm{TeV}$ versus the upper limit of $13.5 \mathrm{MeV}$, tops, for an axion (it is usually a lot LESS) as reported by A. Bischoff-Kim, M. Montgomery and D. Winget ${ }^{11}$ wrote, "Our analysis yields strong limits on the DFSZ axion mass. Our thin hydrogen solutions place an upper limit of $13.5 \mathrm{MeV}$ on the axion, while our thick hydrogen solutions relax that limit to $26.5 \mathrm{MeV}$ ”. For this result, I am picking the $13.5 \mathrm{MeV}$ as the upper limit for axion mass analysis. I.e. values as low as $1 \mathrm{eV}$ have been figured as to axion mass, $5 \mathrm{TeV}$ corresponds to $5.0 \times 10^{12}$ electron volts, Whereas $13.5 \mathrm{MeV}$ is = 13500000 electron volts At the high of the energy scale for axions, there is still roughly $10^{5}-10^{6}$ times more energy in a DM from KK gravitons, as opposed to axions,. Contrasting this with the $400 \mathrm{GeV}$ value for WIMPS specified as of being $400000000000 \mathrm{eV}$, then it is that the KK graviton would yield a far higher amount of energy mass value than the WIMP. The implication may be that eqn (6) has a stronger change in momentum contribution as to the dark matter ram jet / rocket problem, than expected. ${ }^{7}$

Now that the preliminary discussion of the DM ram jet has been brought up, we need to discuss as to what is the space environment as to the ram jet. What would be the DM concentration we could expect? That is not so simple as people think. Key to getting to it would be determining if the present local group of galaxies and star clusters we are in is a local void, or that DM plays the role we hope it does in galaxy formation.

\section{Controversies Of DM/ DE Applications To Cosmology.}

What to consider is the 'cosmic void hypothesis' ${ }^{\text {'7 }}$. See Clifton,. Ferreira and Kate Land ${ }^{12}$. I.e. Clifton raises the following question- can HFGW and detectors permit cosmologist to get to the bottom of this? "Solving Einstein's equations for an averaged matter distribution is NOT the same as solving for the real matter distribution and then averaging the resultant geometry"(to paraphrase their procedure, "We average, then solve when in effect we should solve, then average") .Next, let us look at a recently emerging conundrum of DM feeding into the structure of new galaxies and their far earlier than expected development, i.e. 5 billion years after the big bang. What could cause the earlier clumping? . First of $\mathrm{all}^{7}$, note the formula of variation of DM density which exists has a Hubble parameter $H$, and also the $2^{\text {nd }}$ derivative of the gravitational potential $\nabla^{2} \Phi$, where $\rho_{0}, a_{0}$ are today's values for density and 'distance.' Note, if the Hubble parameter and Friedman equation is 
$H^{2}=\left(\frac{\dot{a}}{a}\right)^{2}=\left[\left(\frac{\rho}{3 M_{4}^{2}}+\frac{\Lambda_{4}}{3}+\frac{\rho^{2}}{36 M_{\text {Planck }}^{2}}\right)-\frac{\kappa}{a^{2}}+\frac{C}{a^{4}}\right] \underset{\substack{\kappa \rightarrow 0, \Lambda_{4} \rightarrow 0}}{\longrightarrow}\left[\frac{\rho}{3 M_{4}^{2}}+\frac{\rho^{2}}{36 M_{\text {Planck }}^{2}}+\frac{C}{a^{4}}\right], \quad$ as well as $\rho \rightarrow \rho(z) \equiv \rho_{0} \cdot(1+z)^{3}-\left[\frac{m_{g}}{8 \pi G}\right] \cdot\left(\frac{a_{0}^{4}}{14 \cdot(1+z)^{4}}+\frac{2 a_{0}^{2}}{5 \cdot(1+z)^{2}}-\frac{1}{2}\right)$, and $^{1+z}=a_{0} / a$, then the contribution of large $z$, i.e. large contributions from red shift, that a significant early contributions will be for non zero contributions from $1 / \rho^{\beta}$ terms, for [ large number l $>\beta \geq 1$ in the DM density variation parameters. So long as $m_{\text {graviton }} \neq 0$, even if $m_{\text {graviton }}$ is very small. In addition, if the following is true, namely having a non linear contribution to the gravitational potential, and $\Phi_{L}$ as linear. This leads to the following iterated formula

$$
\left.\Phi \equiv \Phi_{L}+f_{N L} \cdot \mid \Phi_{L}^{2}-\left\langle\Phi_{L}^{2}\right\rangle\right]+g_{N L} \cdot \Phi_{L}^{3}
$$

For the actual formula, $\nabla^{2} \Phi$ consider the contributions to the expression $f_{N L}$. To do this consider what Verde et al. ${ }^{13}$ put up about $\Phi$ considered to be the gravitational potential, and $\Phi_{L}$ its linear Gaussian contribution. Chingabam, and. Park ${ }^{14}$ used $-4<f_{N L}<80$ at a confidence level of $95 \%$. Now for some sort of bounds as to what may be acceptable bounds in error, based upon CMB data

$$
\left|f_{N L} \cdot\left[\Phi_{L}^{2}-\left\langle\Phi_{L}^{2}\right\rangle\right] \leq 10^{-5} \cdot\right| f_{N L} \mid<\text { ? up to } 10^{-3}
$$

Depending upon which model is used for describing $\Phi_{L}$ i.e. as a perturbation of a gravitational potential, this eqn. (8) may allow us to obtain a good guess as to what dimensions are crucial for the formation of a graviton, i.e. how much spread may be permitted. Also, White and $\mathrm{Hu}^{15}$ have a way to link the gravitational potential $\Phi$ to temperature fluctuations, and do it as

$$
\left.\frac{\Delta T}{T}\right|_{\text {Final }}-\left.\frac{\Delta T}{T}\right|_{\text {Initial }}=-\Phi_{\text {Initial }}
$$

A simple way to understand eqn (9) is to consider if it is linkable to the Sach-Wolfe effect. Here, the Sachs-Wolfe effect (ISW) occurs when the Universe is dominated in density by something other than matter. If the Universe is dominated by matter, then large-scale gravitational potential wells and hills do not evolve significantly. If the Universe is dominated by radiation, or by dark energy, those potentials do evolve, subtly changing the energy of photons passing through them. If so is there a difference in the initial and final ratios $\Delta T / T$ of temperature variations are for different red shift values? Look at

$$
(\delta T / T) \cong(1 / 3) \cdot\left[\Phi_{L}+f_{N L} \cdot\left(\Phi_{L}^{2}-\left\langle\Phi_{L}\right\rangle^{2}\right)\right]
$$

The choice of temperature variations would impact structure formation, and perhaps upon the level of DM contributions to structure even as locally expected for travel to different stars. Beckwith ${ }^{7}$ has written how this issue impacts galaxy formation, but it may have enormous consequences as to DM concentration about our star as well. . On the local stellar level Let us now consider what would be high level DM masses which 
may be appropriate for the DM ram jet. This is important for two reasons. First of all is the supposition that masses above the traditional WIMP mass for DM may contribute to more efficient

The modified KK tower for gravitons will be our candidate for DM ${ }^{7}$.

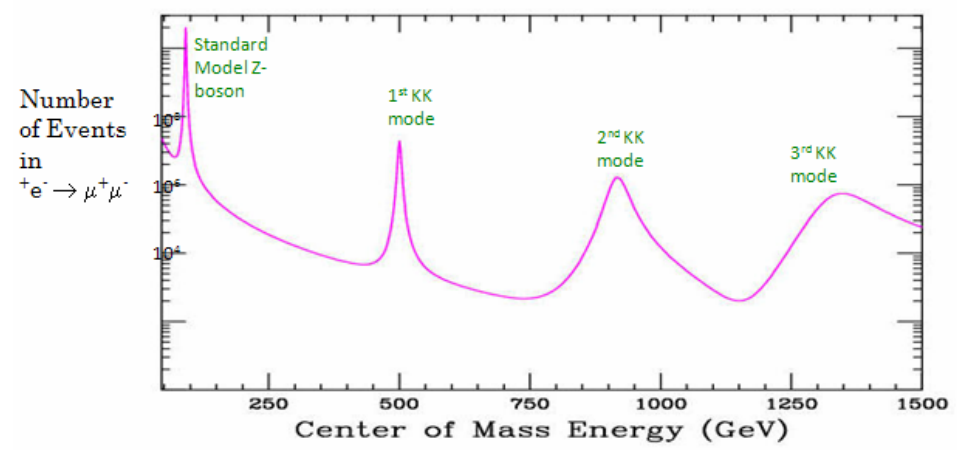

FIGURE 2: Number of Events in $\mathrm{e}+\mathrm{e}-\mathrm{m} \rightarrow \mu+\mu$ - For a conventional braneworld model with a single curved extra dimension of size $\sim 10-17 \mathrm{~cm}$ Numbers range from $10^{4}$ to about $10^{8}$ for the number of events in scattering. First peak is for KK zero modes, a.k.a. the standard Z. boson, ending with the $4^{\text {th }}$ peak for the $3^{\text {rd }} \mathrm{KK}$ mode,

\section{Unanswered Questions and Suggestions for Future Research Endeavors}

First of all, what can researchers expect if KK gravitons exist, and exist in inter stellar space with axions? Cembranos, Feng and Strigari, ${ }^{16}$. (2007) give a partial answer. It is not just the gamma ray spectrum which may be altered. I.e. Boyarsky, Lesgourgues, Ruchaysky and Viel ${ }^{17}$ (2009) have strict Bayesian statistical limits as to what sort of warm to cold dark matter mixes are allowed. One of their basic result, which is put here, $\rho_{\text {Baryons }}, \rho_{\text {Cold-Dark-Matter }}, \rho_{\text {Warm-Dark-Matter }}$ refer to density profiles, of the respective baryons, CDM, and WDM candidates, whereas, the density fluctuations $\delta_{\text {Baryons }}, \delta_{\text {Cold-Dark-Matter }}, \delta_{\text {Warm-Dark-Matter }}$ are with regards to the fluctuations of these density values. So then the density variation $\left(\frac{\delta \rho}{\rho}\right) \equiv \frac{\rho_{\text {Baryons }} \delta_{\text {Baryons }}+\rho_{\text {Cold-Dark-Matter }} \delta_{\text {Cold-Dark-Matter }}+\rho_{\text {Warm-Dark-Matter }} \delta_{\text {Warm-Dark-Matter }}}{\rho_{\text {Baryons }}+\rho_{\text {Cold-Dark-Matter }}+\rho_{\text {Warm-Dark-Matter }}}$

If axions are CDM, and $\mathrm{KK}$ gravitons are for WDM, then up to a point, $\rho_{\text {Warm-Dark-Matter }}$ would dominate eqn. (11) in earlier times, i.e. up to $\mathrm{Z} \sim 1000$. However, Boyarsky, ${ }^{14}$ et al also stress that as of the recent era, i.e. probably for $\mathrm{Z} .55$ to $\mathrm{Z} 0$ today, they would expect to see the following limiting behavior

$$
\begin{aligned}
& \delta_{\text {Baryons }} \equiv \delta_{C D M}, \\
& \delta_{W D M}<<\delta_{C D M}
\end{aligned}
$$

In earlier times, what is put in, with regards to eqn. (11) would be probably far different. However, up in present era, the denominator of eqn (11) would be 
dominated by KK DM, whereas there would be rough equality in the contributions $\rho_{\text {Cold-Dark-Matter }} \delta_{\text {Cold-Dark-Matter }}, \rho_{\text {Warm-Dark-Matter }} \delta_{\text {Warm-Dark-Matter }}$ with the baryon contribution to the numerator being ignorable, due to how small baryon values would be for $\mathrm{Z} \sim .55$ to $\mathrm{Z} \sim 0$ today. Somehow, contributions as to eqn (11) should be compared with.

$$
\left(\frac{\delta \rho}{\rho}\right)_{\text {Horizon }} \cong \frac{k^{3 / 2}\left|\delta_{k}\right|}{\sqrt{2} \pi} \propto \frac{k^{(3 / 2)+3 \alpha-3 / 2}}{\sqrt{2} \pi} \approx(1 / \sqrt{2} \pi) \cdot k^{3 \alpha}
$$

Where $-.1<\alpha<0.2$, and $\alpha \equiv 0 \Leftrightarrow n_{s} \equiv 1$ and to first order, $k \cong H a$. The values, typically of $n_{s} \neq 1$ If working with $H^{2}=\left(\frac{\dot{a}}{a}\right)^{2}=\left[\left(\frac{\rho}{3 M_{4}^{2}}+\frac{\rho^{2}}{36 M_{\text {Planck }}^{2}}\right)+\frac{C}{a^{4}}\right]$, and with a density value $\rho \equiv \rho_{0} \cdot\left(\frac{a_{0}}{a}\right)^{3}-\left[\frac{m_{g} c^{6}}{8 \pi G \hbar^{2}}\right] \cdot\left(\frac{a^{4}}{14}+\frac{2 a^{2}}{5}-\frac{1}{2}\right)$ where $m_{g} \approx 10^{-65}$ grams, and $\alpha<0.2$ is usually picked to avoid over production of black holes, a complex picture emerges. Furthermore, $\alpha<0.2$ and $\alpha \neq 0$. The following limits as of eqn. (11) in early and later times should be reconciled with.

$$
\left(\frac{\delta \rho}{\rho}\right)_{\text {Horizon }} \cong(1 / \sqrt{2} \pi) \cdot k^{3 \alpha} \sim \frac{H^{2}}{\dot{\phi}} \propto 10^{-4}-10^{-5}
$$

The above equation gives inter relationships between the time evolution of a pop up inflaton field $\phi$, and a Hubble expansion parameter $\mathrm{H}$, and a wave length parameter $\lambda=(2 \pi / k) \cdot a(t)$ for a mode given as $\delta_{k}$. What should be considered is the inter relation ship of eqn (14) and $\lambda \leq H^{-1}$. What Beckwith thinks is

$$
\left(\frac{\delta \rho}{\rho}\right) \cong A k^{\left(\frac{n_{s}-1}{2}\right)} \propto 10^{-4}-10^{-5}
$$

Understanding eqn.(8) to eqn. (12) may allow us to understand how to map out concentrations of DM which would be appropriate for our usage once we get to at least the Kuiper belt of the frontiers of the solar system.

\section{Conclusion}

Looking at the KK graviton as an enabler to adding more momentum kick to eqn (4) seems to be a reasonable thought experiment. Of greater concern is the relative distribution of mass/ DM distributions as presented in eqn (11) That has huge implications as to what concentration of DM/ energy scoop up could be configured as to an interstellar probe. Left unsaid here is the necessary datum of a suitable power boost of a ram net, to sufficient speed to work at all. Ultimately, that involves lasers In addition; the density profile of DM and of fuel to the rocket engine has to be mapped out. WMAP techniques will not get that for us. Unfortunately, like many scientific endeavors, it will require test flights in the solar system itself, and not just theory to obtain realistic data as to what to expect. 
Before any of this can be done, i.e. to determine eqn (6) above, space tests to determine which of the three eqn. 3 to eqn. 5 should be used, by space tests in near earth orbit. After that is accomplished, studies as to the efficiency of L4 to L5 laser power requirements need to be initiated. The entire line of inquiry will be determined and started by appropriate engineering tests as to which of the laser force equations is best appropriate.

Finally, appropriate determination of the appropriateness of eqn. (3) to eqn (5) will lead to technology development which will make realistic exploitation of the local solar system a technological feasible project. Having flights say up to Jupiter, and the asteroid belt shortened to say three months to half a year for commercial space craft will have much the same effect upon the Earths economy, and the image of our own species, homo sapiens, as did the age of exploration had on continental Europe from the $16^{\text {th }}$ to $19^{\text {th }}$ centuries AD.

\section{ACKNOWLEDGMENTS}

First is to thank Andrew Pakhomov, for allowing me to join and partake of AIBEP.org. Many thanks, Andrew and I hope to in the years ahead to make his investment in my participation worth while to all concerned. Secondly , is to thank both Amara Angelica and also H. Keith Henson, Founder, L5 Society for a back ground briefing as of surface to earth orbit physics boosts via lasers, a subject I am very interested in, as well. Also, Eric Davis whom over the years has corrected many of my initial assumptions and pointed me in more nuanced and focused /'fruitful venues of scientific inquiry.

\section{REFERENCES}

1 L. Roszkowski, (private communications) with A. W..Beckwith at 12 Marcel Grossman meeting, July 17th, 2009, in Paris observatory, during the Dark matter section of the conference, in Parallel section run by Dr. Chardin on Dark Matter

2 A.W. Beckwith, "Cosmic Deceleration Parameter Q(Z) Dependence Upon Gravitons? Implications for the DM Rocket/ram Jet Mode”, talk in SPESIF, 2010, February , http://www.vixra.org/abs/0910.0011

3 H. Keith Henson, Founder, L5 Society( private communications)

4 A.W. Beckwith. Created by Author in October 2009

5 V. Sherstobitov, N.. Kaliteevskiy, V.. Kuprenyup, et al. , "Computer simulations of a solid state Laser System for Propulsion of a Space “Tugboat” from LEO to GEO” pages , AIP Conference proceedings 766, 2004 , pp 347-3

6 M. G. Millis, "Pre requisites for Space Drive Science", pages 137-171; from "Frontiers of Propulsion Science”, Editors M.G. Millis and E. Davis, volume 227 of Progress in Asronautics and Aeronautics, AIAA, Reston, Virginia, USA 2009 
7 Y. Satov, Y. Sharkov, Y. Smakovski, K. Makarov,. et al. ,” The “SKATE” CO2 Gigawatt Laser for a Laser-Plasma Generator of Ions and Nuclei”, The Russsian journal of Laser physics research,,Volume 25, Number 6 / November, 2004

8 National Aeronautics and Space Administration Small Business Technology Transfer (STTR)

Program 2002 Phase 2 Proposals Selected for Negotiation of Contracts. See Phillip Battle (406) 522-

0388 Montana State University Physics Dept. EPS 264 Bozeman , MT 59717-3840 02-2-020055

GSFC Ultra-Stable Miniature Seed Laser for High Power Nd:YAG Lasers , http://sbir.nasa.gov/SBIR/sttr2002/phase2/awards/02topic.html.

9 A.W. Beckwith,” Hypothetical Dark Matter/ axion Rockets: Dark Matter in Terms of Space Physics Propulsion”, AIP Conf.Proc.1103:276,2009., with another version at http://vixra.org/abs/0909.0050

10 P. Sikivie, PRL 51, 1415 (1983)

11 A. Bischoff-Kim., H. Montgomery, \&. D. Winget ., "Strong Limits on the DFSZ Axion Mass with G117-B15A,” ApJ, 675, 1512 , 2008

12 Timothy Clifton, Pedro G. Ferreira and Kate Land,” Living in a Void: Testing the Copernican

Principle with Distant Supernovae”, Phys. Rev. Lett. 101 (2008) 131302,

http://arxiv.org/PS_cache/arxiv/pdf/0807/0807.1443v2.pdf

13 Licia Verde, Alan Heavens, Sabino Matarrese, "Projected bispectrum in spherical harmonics and its application to angular galaxy catalogues”, MNRAS, 318

14 P. Chingabam, C. Park P. Chingabam, C. Park;”Statistical nature of Non Gaussianity from cubic order Primoridal perturbations : CMB map simulations and genus statistics” http://arxiv.org/PS_cache/arxiv/pdf/0908/0908.1696v1.pdf

15 W. Martin, and W, Hu, The Sachs-Wolfe effect. Astronomy and Astrophysics 321, 89. 1997

16 J. Cembranos, J.. Feng and L. Strigari,” Dark Matter Decaying Now”, Contributed to XXIII International Symposium on Lepton and Photon Interactions at High Energy, Aug 13-18, 2007, Daegu, Korea Report No. S3-4-228, ABS-S3-4-013; arXiv:0708.0239

17 A, Boyarsky, J. Lesgourgues, O. Ruchaysky and M. Viel,” Realistic sterile neutrino dark matter with keV mass does not contradict cosmological bounds”, Phys.Rev.Lett.102:201304,2009,

arXiv:0812.3256 (December 2008) 